\title{
Identification and Stochastic Optimizing the UAV Motion Control in Turbulent Atmosphere
}

\author{
Yevgeny Somov ${ }^{1 *}$, Nikolay Rodnishchev ${ }^{2}$, Tatyana Somova ${ }^{3}$ \\ ${ }^{1}$ Samara State Technical University, Samara, Russia \\ e_somov@mail.ru - (D) 0000-0002-7998-6575 \\ ${ }^{2}$ Kazan National Research Technical University - KAI, Kazan, Russia \\ nrodnishev@yandex.ru - (D) 0000-0001-5901-7796 \\ ${ }^{3}$ Samara State Technical University, Samara, Russia \\ te_somova@mail.ru - (DD 0000-0002-1862-7210
}

\section{Keywords}

\section{Abstract}

In a class of diffusion Markov processes, we formulate a problem of identification of nonlinear stochastic dynamic systems with random parameters, multiplicative and additive noises, control functions, and the state vector at a final time moment. For such systems, the identifiability conditions are being studied, and necessary conditions are formulated in terms of the general theory of extreme problems. The developed engineering methods for identification and optimizing nonlinear stochastic systems are presented as well as their application for unmanned aerial vehicles under wind disturbances caused by atmospheric turbulence, namely, for optimizing the autopilot parameters during a rotary maneuver of an unmanned aerial vehicle in translational motion, taking into account the identification of its angular velocities.

\author{
Unmanned Aerial Vehicle \\ Motion Control \\ Identification \\ Stochastic Optimization
}

\section{Time Scale of Article}

Received 29 September 2021 Revised until 13 October 2021 Accepted 20 October 2021 Online date 24 December 2021

\section{Introduction}

Stochastic differential and discrete models are applied in the study of complex controlled systems under conditions of random parametric, structural, and external disturbances.

The mathematical foundations for such researches are presented in well-known monographs by Bulinsky and Shiryaev, 2005; Evlanov and Konstantinov, 1976; Fleming and Rishel, 1975; Gikhman and Skorokhod, 1977; Kazakov, 1977; Oksendal, 2000; Solodov and Solodov, 1988 et al. . Here and further, we apply for the alphabetical citation order.

Strict mathematical methods for optimizing nonlinear systems are also known; for example, Dubovitskii and Milyutin, 1965; Girsanov, 1970; Ioffe and Tikhomirov,
1974; Kazakov and Artemyev, 1980; Kolosov, 1984 et al..

In the applied theory of optimizing nonlinear stochastic systems, approximate methods based on parametric or functional approximation of the a posteriori probability distribution density are used. Parametric approximation methods are applied to determine the characteristics of stochastic processes, namely, a posteriori moments or cumulants, which are usually called semi-invariants, see the monographs by Bodner et al., 1987; Chernetsky, 1968; Denisov and Rodnishchev, 2017; Dostupov, 1970; Kozhevnikov, 1978; Malakhov, 1978; Potseluyev, 1984; Pugachev and Sinitsyn, 1985 et al..

Our theoretical results on this topic were presented in research papers (Rodnishchev, 2001a,b; Rodnishchev and Khairullin, 2010). These results were implemented in the Russian aerospace industry, namely in control

*: Corresponding Author Yevgeny Somov, e_somov@mail.ru

DOI: 10.23890/IJAST.vm02is02.0202 
systems of spacecraft (Rodnishchev et al., 2019), including mini-satellites for communication (Fig. 1) and the Earth-observing (Fig. 2), space robot-manipulators (SRMs), Fig. 3 (Somov et al. 1., 2019), as well as Russian passenger airliners, Fig. 4 (Rodnishchev and Somova, 2019), in control systems of turboprop engines for various aviation equipment (Bodner et al., 1987; Kozhevnikov et al., 1989).

In this paper, methods for identification in stochastic control systems and study the problem of optimizing parameters of unmanned aerial vehicle (UAV) autopilot during its turning maneuver in translational movement under stochastic atmospheric turbulence are briefly presented.

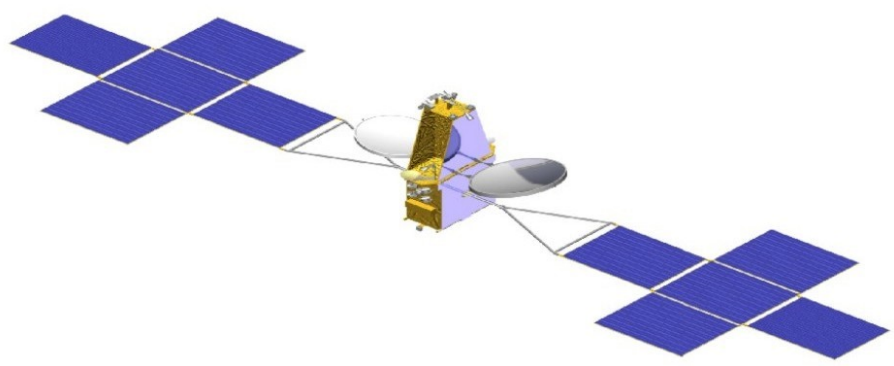

Fig. 1. The communication mini-satellite

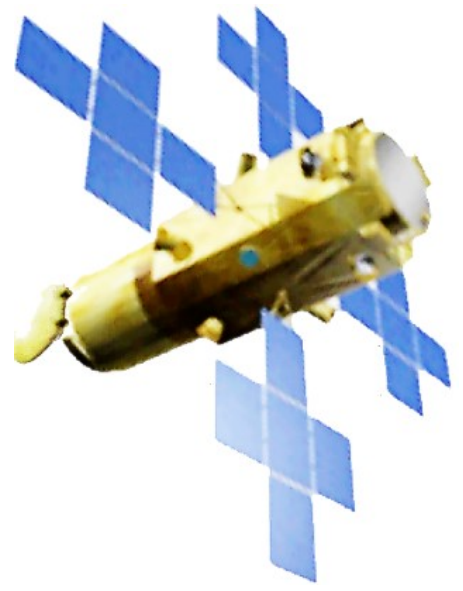

Fig. 2. The Earth-surveying mini-satellite

\section{Models and the Problem Statement}

In this section, the problem of identifying vectors of parameters $\mathbf{a} \equiv\left\{a_{i}\right\} \in \mathrm{R}^{m}$ and control $\mathbf{u} \equiv\left\{u_{i}\right\} \in \mathrm{R}^{r}$ is studied for nonlinear stochastic system

$$
\begin{aligned}
d y_{i} & \left.=\left(\sum C_{i j}(t, \mathbf{b}) d t+d w_{i j}(t)\right) \phi_{i j}(t, \mathbf{y}, \mathbf{b}, \mathbf{u}, \mathbf{a})\right) \\
& +\sum \sigma_{i j}(t, \mathbf{y}, \mathbf{b}) d \eta_{j}(t), i \in \mathrm{N}_{1}^{n}, \quad t \in\left[t_{\mathrm{i}}, t_{\mathrm{f}}\right]
\end{aligned}
$$

with a state vector $\mathbf{y}=\left\{y_{i}\right\} \in \mathrm{R}^{n}$ at initial condition $\mathbf{y}_{\mathrm{i}}=\left\{y_{\mathrm{i} i}\right\}$ where $\mathrm{N}_{1}^{n} \equiv[1,2, \ldots n]$ and observations

$$
z_{k}=\sum c_{k v} y_{v}+\dot{w}_{k}(t), k \in \mathrm{N}_{1}^{p} .
$$

Identification efficiency is evaluated by functional minimum

$$
I_{0}(\mathbf{u}, \mathbf{a})=\int_{t_{\mathrm{i}}}^{t_{\mathrm{f}}} E\left[\sum \alpha_{k}\left(z_{k}-\sum c_{k v} y_{v}\right)^{2}\right] d t
$$

and control objectives, technical and operational requirements to the system are determined by constraints on the final system status of the equality type

$$
I_{s}(\mathbf{u}, \mathbf{a})=E\left[f_{s}\left(\mathbf{y}_{\mathrm{f}}\right)\right]-c_{s}=0, \quad s \in \mathrm{N}_{1}^{q} .
$$

Here $E[\cdot]$ is the expectation operator, $t_{\mathrm{i}}$ and $t_{\mathrm{f}}$ are the initial and final points of a time interval and $\mathbf{y}_{\mathrm{f}}=\left\{y_{\mathrm{fi}}\right\}=\mathbf{y}\left(t_{\mathrm{f}}\right)$.

As a control vector $\mathbf{u}(\cdot)$, we study a program control $\mathbf{u}(t)$ or feedback control $\mathbf{u}(t, \mathbf{y})$. Control $\mathbf{u}(t)$ is determined on the set $\left.\mathrm{S}=\left\{\mathbf{u}(t) \in L_{2}\left[t_{\mathrm{i}}, t_{\mathrm{f}}\right]: \mathbf{u}(t)\right\} \in U, t \in\left[t_{\mathrm{i}}, t_{\mathrm{f}}\right]\right\}$, where $L_{2}\left[t_{\mathrm{i}}, t_{\mathrm{f}}\right]$ is the space of measurable functions with quadratic metrics and $U \subset \mathrm{R}^{r}$ is a convex set.



Fig. 3. The SRM is preparing to capture a failed satellite

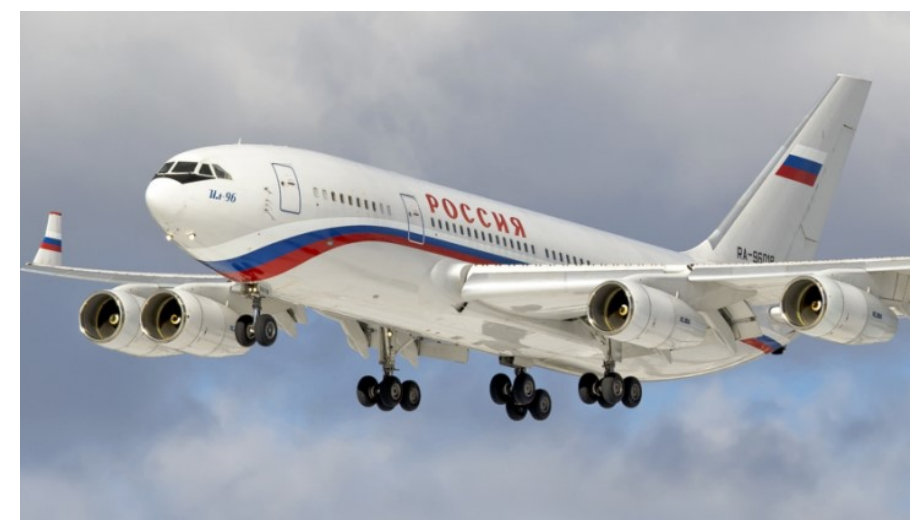

Fig. 4. Russian passenger airliner IL 96-300 on landing

The feedback control $\mathbf{u}(t, \mathbf{y})$ is considered as Borelian function as either a random element in $L_{2}$ or a nonanticipating process relative to Wiener processes $w_{i j}, \eta_{j}$ with values in $U$.

Next, column a determinate the controlling parameters; $\mathbf{b} \equiv\left\{b_{i}\right\} \in \mathrm{R}^{l}$ is a random vector; $w_{i j}(t)=W_{i j}(t) d t$ and $\eta_{j}(t)=\mathrm{N}_{j}(t) d t$ are Stratonovich stochastic differentials of Wiener processes $w_{i j}(t)$ and $\eta_{j}(t)$, moreover, the process $w_{i j}(t)$ describes multiplicative noises affecting 
the system, the process $\eta_{j}(t)$ describes additive noises, $W_{i j}(t)$ are white Gaussian noises that describes random internal perturbations common for the system, $\mathrm{N}_{j}(t)$ are the white Gaussian noises; $C_{i j}(t, \mathbf{b}), \phi_{i j}(t, \mathbf{y}, \mathbf{b}, \mathbf{u}, \mathbf{a})$ and $\sigma_{i j}(t, \mathbf{y}, \mathbf{b})$ are given nonrandom functions satisfying given requirements of solution existence (1), and $\mathbf{z} \equiv\left\{z_{k}\right\} \in \mathrm{R}^{p}$ is observed vector of tester coordinates $z_{k}, k \in \mathrm{N}_{1}^{p}, p \leq n$. The matrix component $\left(c_{k v}\right)$ determines the selection of observed system coordinates $(1), \dot{w}_{k}(t)$ is derivative of Wiener process, $w_{k}(t)$ is component $R_{k}(t)$ of tester additive white noise.

At last, $I_{s}(\mathbf{u}, \mathbf{a})$ are continuous and continuously differentiable functionals on a set of variables, and $I_{0}(\mathbf{u}, \mathbf{a})$ is bounded functionally differentiable on the set of variables and $\alpha_{k}$ are the weight numbers.

At accepted conditions concerning the right parts, solution (1) exists, and it is unambiguous. However, this solution does not need to be a Markov process. That is why, to make (1) describe the Markov process, we introduce an extended state vector $\mathbf{x} \equiv\left\{x_{i}\right\}=\{\mathbf{y}, \mathbf{b}\}$. Relative to the state vector $\mathbf{x}$ equations (1) reduce themselves to an equivalent system of diffusion stochastic differential equations

$$
\begin{aligned}
& d x_{i}\left.=\left(\sum C_{i j}(t, \mathbf{x}) d t+d w_{i j}(t)\right) \phi_{i j}(t, \mathbf{x}, \mathbf{u}, \mathbf{a})\right) \\
&+\sum \sigma_{i j}(t, \mathbf{x}) d \eta_{j}(t), \\
& d x_{i}=0, \quad x_{i}\left(t_{\mathrm{i}}\right)=y_{\mathrm{i} i} \forall i \in \mathrm{N}_{1}^{n} ; \\
& x_{i}\left(t_{\mathrm{i}}\right)=b_{i} \quad \forall i \in \mathrm{N}_{n+1}^{n+l} .
\end{aligned}
$$

These equations have a single solution and describe the diffusion Markov process $\forall t \in\left[t_{\mathrm{i}}, t_{\mathrm{f}}\right]$; the posterior density of state probability $p(t, \mathbf{x} \mid \overline{\mathbf{z}})$ satisfies the Stratonovich-Kushner equation (a generalization of the well-known Fokker-Planck- Kolmogorov equation)

$$
\partial p(\cdot) / \partial t=L(\cdot) p(\cdot)+\left(F(\cdot)-\int F(\cdot) p(\cdot) d \mathbf{x}\right) p(\cdot)
$$

with $\mathbf{x} \in \Omega \subset \mathrm{R}^{n}$ and initial condition $\left.p(t, \mathbf{x} \mid \overline{\mathbf{z}})\right|_{t=t_{\mathrm{i}}}=p\left(t_{\mathrm{i}}, \mathbf{x}\right)$ . Here $F(\cdot)=F(\mathbf{x}, \mathbf{z})$ and $L(\cdot) p(\cdot)=L(t, \mathbf{x}, \mathbf{u}, \mathbf{a}) p(t, \mathbf{x} \mid \overline{\mathbf{z}})$ is an elliptic operator determined by the formula

$$
L(\cdot) p(\cdot)=-\sum \partial\left[A_{i} p(\cdot)\right] / \partial x_{i}+(1 / 2) \sum \partial^{2}\left[B_{i p} p(\cdot)\right] / \partial x_{i} \partial x_{p}
$$

with coefficients of the drift $A_{i}=A_{i}(t, \mathbf{x}, \mathbf{u}, \mathbf{a})$ and diffusion $B_{i p}=B_{i p}(t, \mathbf{x}, \mathbf{u}, \mathbf{a})$. In the density function, $p(t, \mathbf{x} \mid \overline{\mathbf{z}})$ the vector $\overline{\mathbf{z}}$ means that the whole output signal realization of the tester observed on the time interval $t \in\left[t_{\mathrm{i}}, t_{\mathrm{f}}\right]$ is applied.

Assume that $G_{p k}$ are the mutual forces of tester noises and $\quad F(\mathbf{x}, \mathbf{z})=\sum\left(c_{p q} x_{q} / G_{p k}\right)\left(z_{k}-(1 / 2) \sum c_{k v} x_{v}\right) \quad$ scalar function characterize the tester properties. According to a theory of Markov processes, original identification problem (1) - (3) concerning the extended vector of system state $\overline{\mathbf{x}}=\left\{\mathbf{x}, x_{n+1}\right\}$ reduces itself to an equivalent terminal problem with distributed parameters relative to a posterior density function $p(t, \overline{\mathbf{x}} \mid \overline{\mathbf{z}})$ in the form

$$
\begin{aligned}
& I_{0}(\mathbf{u}, \mathbf{a})=\int x_{n+1} p\left(t_{\mathrm{f}}, \overline{\mathbf{x}} \mid \overline{\mathbf{z}}\right) d \overline{\mathbf{x}} \Rightarrow \min \\
& \partial p(\cdot) / \partial t=\bar{L}(\cdot) p(\cdot)+\left(F(\cdot)-\int F(\cdot) p(\cdot) d \overline{\mathbf{x}}\right) p(\cdot)
\end{aligned}
$$

with $F(\cdot)=F(\overline{\mathbf{x}}, \mathbf{z})$ and the condition $\left.p(t, \overline{\mathbf{x}} \mid \overline{\mathbf{z}})\right|_{t=t_{\mathrm{i}}}=p\left(t_{\mathrm{i}}, \overline{\mathbf{x}}\right)$;

$$
I_{s}(\mathbf{u}, \mathbf{a})=\int f_{s}(\mathbf{x}) p\left(t_{\mathrm{f}}, \overline{\mathbf{x}} \mid \overline{\mathbf{z}}\right) d \overline{\mathbf{x}}-c_{s}=0, \quad s \in \mathrm{N}_{1}^{q} .
$$

Here the component $x_{n+1}(t)$ is determined by equation

$$
d x_{n+1}=\sum \alpha_{k}\left(z_{k}-\sum c_{k v} x_{v}\right)^{2} d t \equiv g(\mathbf{x}) d t
$$

with initial condition $x_{n+1}\left(t_{\mathrm{i}}\right)=0$ and the operator

$$
\bar{L}(\cdot) p(\cdot) \equiv L(\cdot) p(\cdot)-\partial[g(\mathbf{x}) p(t, \overline{\mathbf{x}} \mid \overline{\mathbf{z}})] / \partial x_{n+1} \cdot
$$

This model is identifiable if the system (5), (11) is controllable (Rodnishchev, 2001a; Denisov and Rodnishchev, 2017).

The main objectives of this paper are a brief presentation of our general approach to the considered problem and its practical application to optimizing the UAV autopilot parameters at its route turning under the turbulent wind perturbations.

\section{Method and the Approach}

For obtaining the necessary identification conditions in terms of conjugate cones in the general theory of extreme problems (Dubovitskii and Milyutin, 1965; Ioffe and Tikhomirov, 1974), the method by Girsanov (1970) is applied.

In this case, the solution to the equation (6) is needed as well as the coupled Bellman parabolic equation. However, as it is known, analytic solutions to linear and special cases of nonlinear stochastic systems only can be obtained. Here our approach (Rodnishchev, 2001) is applied, which is based on employing mathematical statistics.

The identification problem with respect to a posteriori semi invariant is reduced to the equivalent extreme problem of the estimation parameters, control, and components of the state vector in the form

$$
\begin{gathered}
\omega_{1}^{n+1}\left(t_{\mathrm{f}}\right) \Rightarrow \min , \\
\dot{\omega}_{1}^{n+1}=E\left[\sum \alpha_{k}\left(z_{k}-\sum c_{k v} x_{v}\right)^{2}\right], \quad \omega_{1}^{n+1}\left(t_{\mathrm{i}}\right)=0 ;
\end{gathered}
$$




$$
\begin{aligned}
\dot{\omega}_{1}^{j}= & E\left[A_{j}(\cdot)\right]+E\left[x_{j} F(\mathbf{x}, \mathbf{z})\right]-\omega_{1}^{j} E[F(\mathbf{x}, \mathbf{z})] \equiv g_{1}^{j}, \\
\dot{\omega}_{11}^{j p}= & E\left[\left(x_{j}-\omega_{1}^{j}\right) A_{p}+\left(x_{p}-\omega_{1}^{p}\right) A_{j}+E\left[B_{j p}(\cdot)\right]\right. \\
+ & E\left[\left(x_{j}-\omega_{1}^{j}\right)\left(x_{p}-\omega_{1}^{p}\right) F(\mathbf{x}, \mathbf{z})\right]-\omega_{11}^{j p} E[F(\mathbf{x}, \mathbf{z})], \\
\dot{\omega}_{2}^{j}= & 2 E\left[\left(x_{j}-\omega_{1}^{j}\right) A_{j}(\cdot)\right]+E\left[B_{j j}(\cdot)\right] \\
+ & E\left[\left(x_{j}-\omega_{1}^{j}\right)^{2} F(\mathbf{x}, \mathbf{z})\right]-\omega_{2}^{j} E[F(\mathbf{x}, \mathbf{z})], \\
& \cdots \cdots \cdots \cdots \ldots . . . \\
\dot{\omega}_{N_{j}}^{j}= & N_{j} E\left[\left(x_{j}-\omega_{1}^{j}\right) A_{j}^{N_{j}-1}(\cdot)\right]+(1 / 2) N_{j}\left(N_{j}-1\right) \\
& E\left[\left(x_{j}-\omega_{1}^{j}\right)^{N_{j}-2} B_{j j}(\cdot)\right]-\sum_{q_{j}=1}^{N_{j}-2} C_{N_{j}}^{q_{j}} g_{q_{j}}^{j} \omega_{N_{j}-q_{j}}^{j} \\
& +E\left[\left(x_{j}-\omega_{1}^{j}\right)^{N_{j}} F(\mathbf{x}, \mathbf{z})\right]-\omega_{N_{j}}^{j} E[F(\mathbf{x}, \mathbf{z})]
\end{aligned}
$$

for $N_{j} \geq 3$ with initial conditions

$\omega_{1}^{j}\left(t_{\mathrm{i}}\right)=c_{1 \mathrm{i}}^{j}, \omega_{11}^{j p}\left(t_{\mathrm{i}}\right)=b_{11 \mathrm{i}}^{j p}, \omega_{2}^{j}\left(t_{\mathrm{i}}\right)=b_{2 \mathrm{i}}^{j}, \ldots, \omega_{N_{j}}^{j}\left(t_{\mathrm{i}}\right)=b_{N_{j} \mathrm{i}}^{j}$

and indexes $j, p \in \mathrm{N}_{1}^{n}$, also taking into account (10).

The equations (13) present variations of semi-invariants $\omega_{1}^{j}$ of the 1-st order by $j$-th components for the system state vector, coinciding with the mathematical expectations; semi-invariants $\omega_{2}^{j}$ and $\omega_{11}^{j p}$ of 2-nd order by $j$-th components and the relationship between $j$-th and $p$-th components, coinciding with the dispersion and correlation functions of the state vector; at last, semi-invariants $\omega_{N_{j}}^{j}$ of the $N_{j}$-th order.

For the closure of a shortened system of differential equations (13) and an approximate representation of the higher moments through the lower moments, the method of moment semi-invariants is applied (Dashevskii, 1976).

Assume $N_{j}=8$, that is sufficient to solve practical problems. Semi-invariants are not independent, and they are bound by the conditions for the functions $f_{\mu}, \mu \in \mathrm{N}_{1}^{9}$ (Malakhov, 1978):

$$
\begin{array}{ll}
f_{1}=2\left(\omega_{2}^{j}\right)^{2}+\omega_{4}^{j} \geq 0 ; & f_{2}=2 \omega_{2}^{j} \omega_{2}^{p}+\omega_{22}^{j p} \geq 0 ; \\
f_{3}=a \omega_{2}^{j}\left(\omega_{2}^{p}\right)^{2}+\omega_{24}^{j p} \geq 0 ; & f_{4}=a\left(\omega_{2}^{j}\right)^{2} \omega_{2}^{p}+\omega_{42}^{j p} \geq 0 ; \\
f_{5}=a\left(\omega_{2}^{j}\right)^{2}+\omega_{6}^{j} \geq 0 ; & f_{6}=b\left(\omega_{2}^{j}\right)^{2}\left(\omega_{2}^{p}\right)^{2}+\omega_{44}^{j p} \geq 0 ; \\
f_{7}=b \omega_{2}^{j}\left(\omega_{2}^{p}\right)^{3}+\omega_{26}^{j p} \geq 0 ; & f_{8}=b\left(\omega_{2}^{j}\right)^{3} \omega_{2}^{p}+\omega_{62}^{j p} \geq 0 ; \\
f_{9}=b\left(\omega_{2}^{j}\right)^{4}+\omega_{8}^{p} \geq 0, \text { where } a=105 \text { and } b=166214 .
\end{array}
$$

These functional inequalities should be performed in the solution of the optimization problem (12), (13) on the time interval $t \in\left[t_{\mathrm{i}}, t_{\mathrm{f}}\right]$, so that is the functions $f_{\mu}$ must belong to an integral variety $f_{\mu} \geq 0$ for the set of differential equations

$$
\left.\left(d f_{\mu}(t) / d t\right)\right|_{(13)}=-\rho f_{\mu}\left[1+\operatorname{sign}\left(f_{\mu}\right)\right] / 2, \quad \mu \in \mathrm{N}_{1}^{9}
$$

with $\rho>0, \operatorname{sign}(0)=0, f_{\mu}\left(t_{\mathrm{f}}\right) \geq 0$ when $f_{\mu}\left(t_{\mathrm{i}}\right)=0 \quad \forall \mu \in \mathrm{N}_{1}^{9}$.

\section{Optimizing the UAV Autopilot Parameters}

This section solves the problem of stochastic optimization of autopilot parameters for the UAV turn mode, taking into account the identification of UAV angular velocities in atmospheric turbulence.

At an angular velocity $\omega_{y}^{\#}=$ const, the UAV lateral angular motion is described in standard notations by the equations

$$
\begin{aligned}
& \dot{\beta}=k_{\gamma} \gamma+k_{\beta}\left(\beta+\beta_{w}\right)+\alpha_{o} \omega_{x}+\omega_{y} ; \\
& \dot{\omega}_{x}=-L_{\beta}\left(\beta+\beta_{w}\right)+L_{\delta} \delta-L_{x} \omega_{x}-L_{y} \omega_{y}-l_{\mathrm{e}} i_{y}^{\mathrm{e}} \omega_{y}^{*} ; \\
& \dot{\omega}_{y}=-N_{\beta}\left(\beta+\beta_{w}\right)-N_{x} \omega_{x}-N_{y} \omega_{y} ; \\
& \dot{\gamma}=\omega_{x} ; \quad \dot{\psi}=\omega_{y} ; \quad \dot{\delta}=\omega_{y}-\omega_{y}^{*} ; \\
& \dot{\beta}_{w}=-q_{x} \beta_{w}+\sigma_{\beta_{w}} \sqrt{2 q_{x}} N(t),
\end{aligned}
$$

where $q_{x}=V_{\mathrm{o}} / L_{w_{x}}$ with a nominal true airspeed $V_{\mathrm{o}}$ of the UAV flight and a scale $L_{w_{x}}$ of turbulence; $\sigma_{\beta_{w}}=\sigma_{\omega_{x}} / V_{\mathrm{o}}$ with the root mean square (RMS) value $\sigma_{\omega_{x}}$ of the turbulence intensity, $N(t)$ is standard white noise, and $\alpha_{\mathrm{o}}$ is the UAV balancing angle of attack. Here we use the following notations

$$
\begin{aligned}
& L_{\beta}=l_{\beta}+l_{\mathrm{e}} i_{\beta}^{\mathrm{e}} ; L_{x}=l_{\omega_{x}}+l_{\mathrm{e}} i_{x}^{\mathrm{e}} ; L_{\delta}=l_{\mathrm{e}} q_{\mathrm{e}} ; L_{y}=l_{\omega_{y}}-l_{\mathrm{e}} i_{y}^{\mathrm{e}} ; \\
& N_{\beta}=n_{\beta}+n_{\mathrm{d}} i_{\beta}^{\mathrm{d}} ; \quad N_{x}=n_{\omega_{x}}+n_{\mathrm{d}} i_{x}^{\mathrm{d}} ; \quad N_{y}=n_{\omega_{y}}-n_{\mathrm{d}} i_{y}^{\mathrm{d}},
\end{aligned}
$$

where $k_{\gamma}, k_{\beta}, l_{\beta}, l_{\omega_{x}}, l_{\omega_{y}}, l_{\mathrm{e}}, n_{\beta}, n_{\omega_{x}}, n_{\omega_{y}}, n_{\mathrm{d}}$ are the UAV aerodynamic coefficients, and $i_{\beta}^{\mathrm{e}}, i_{x}^{\mathrm{e}}, i_{y}^{\mathrm{e}}, q_{\mathrm{e}}, i_{\beta}^{\mathrm{d}}, i_{x}^{\mathrm{d}}, i_{y}^{\mathrm{d}}$ are the autopilot gear ratios.

To determine variations of the additional sliding angle $\beta_{w}$, we use a model for the horizontal wind turbulence components, which describes a Gaussian random process with a spectral density $S(\omega)=(2 / \pi) \sigma_{\omega_{x}}^{2} L_{w_{x}} /\left(1+\omega^{2} L_{w_{x}}^{2}\right)$, and when using the formative filter (Pugachev and Sinitsyn, 1985), it is represented by a stochastic differential equation in (15) with the input noise $N(t)$.

The autopilot gear ratios are linearly related to the coefficients $L_{x}, L_{y}, L_{\delta}, N_{\beta}, N_{x}, N_{y}$ of the stochastic system (15), so the definition of the ratios $i_{\beta}^{\mathrm{e}}, i_{x}^{\mathrm{e}}, i_{y}^{\mathrm{e}}, q_{\mathrm{e}}, i_{\beta}^{\mathrm{d}}, i_{x}^{\mathrm{d}}, i_{y}^{\mathrm{d}}$ is reduced to the optimization of these coefficients with the angular velocity measurements $z_{1}=\omega_{x}+N_{\omega_{x}}, z_{2}=\omega_{y}+N_{\omega_{y}}$, where the vector $\mathbf{z} \equiv\left\{z_{1}, z_{2}\right\} \quad N_{\omega_{x}}$ and $N_{\omega_{y}}$ are standard white noises. 
The effectiveness of the linear control law optimization is estimated the functional minimum

$$
I_{0}=\int_{t_{\mathrm{i}}}^{t_{\mathrm{f}}} E\left[\left(\omega_{y}-\omega_{y}^{\#}\right)^{2}\right] d t \Rightarrow \min .
$$

Since wind disturbances caused by horizontal gusts can lead to large deviations when turning the UAV, the parameters of control law are determined so that coefficients $C_{i}, i \in \mathrm{N}_{1}^{5}$ of the characteristic polynomial for the closed-loop system (15) provide a consistent choice of parameters from the asymptotic stability region.

With the notation, $q \equiv \alpha_{\mathrm{o}} / k_{\gamma}$ this region has the boundary defined by the following constraints:

$$
\begin{aligned}
& L_{x}^{3}-C_{1} L_{x}^{2}+C_{2} L_{x}-\left(C_{3}-C_{4} q+C_{5} q^{2}\right)=0 ; \\
& L_{x}+N_{y}-\left(C_{1}-k_{\beta}\right)=0 ; k_{\gamma} L_{y} N_{\beta}+\left(C_{4}-C_{5}\right) q=0 ; \\
& L_{x} N_{y}+N_{\beta}-\left(C_{2}-k_{\beta}\left(C_{1}-k_{\beta}\right)\right)=0 ; k_{\gamma} L_{\delta} N_{\beta}-C_{5}=0 .
\end{aligned}
$$

To solve the optimization problem, the additional variable $x_{8}(t)$ is introduced, which is determined by the solution of the differential equation

$$
\dot{x}_{8}=\left(\omega_{y}-\omega_{y}^{\#}\right)^{2}
$$

with the initial condition $x_{8}\left(t_{\mathrm{i}}\right)=0$, and the functional (16) is reduced to the following terminal form

$$
I_{0}=E\left[x_{8}\left(t_{\mathrm{f}}\right) \Rightarrow \min .\right.
$$

Thus, the problem of optimizing the autopilot gear ratios is reduced to determining the vector column

$$
\mathbf{a} \equiv\left\{a_{i}, i \in \mathrm{N}_{1}^{6}\right\} \equiv\left\{L_{x}, L_{y}, L_{\delta}, N_{\beta}, N_{x}, N_{y}\right\}
$$

with a providing of the minimum terminal functional (17) and taking into account the need for identification of the angular velocities $\omega_{x}$ and $\omega_{y}$ to the above restrictions on asymptotic stability region.

The system (15) describes a diffusion on Markovian process with the state vector $\mathbf{x}=\left\{x_{i}\right\} \equiv\left\{\beta, \omega_{x}, \omega_{y}, \gamma, \psi, \delta, \beta_{w}\right\}$, the coefficients of drift

$A_{1}=-k_{\beta}\left(x_{1}+x_{7}\right)+\alpha_{0} x_{2}+x_{3}+k_{\gamma} x_{4}$

$A_{2}=-a_{1} x_{2}-a_{2}\left(x_{3}-\omega_{y}^{*}\right)+a_{3} x_{6}-l_{\omega_{x}} \omega_{y}^{\#} ;$

$A_{3}=-a_{4}\left(x_{1}+x_{7}\right)-a_{5} x_{2}-a_{6} x_{3} ; A_{4}=x_{2} ;$

$A_{5}=x_{3} ; A_{6}=x_{3}-\omega_{y}^{\#} ; A_{7}=-q_{x} x_{7} ; A_{8}=\left(x_{3}-\omega_{y}^{*}\right)^{2}$

and diffusion $B_{77}=2 \sigma_{\omega_{x}}^{2} q_{x}$. Here, for identification of the above angular velocities, the scalar function

$F(\mathbf{x}, \mathbf{z})=x_{2}\left(z_{1}-x_{2} / 2\right) / G_{\omega_{x}}+x_{3}\left(z_{2}-x_{3} / 2\right) / G_{\omega_{y}}$

is applied. So, the stochastic problem is reduced to the deterministic one for the semi-invariants as follows

$$
\omega_{8}^{1}\left(t_{\mathrm{f}}\right) \Rightarrow \mathrm{min}
$$

$$
\begin{aligned}
\dot{\omega}_{1}^{j} & =E\left[A_{j}(\cdot)\right]+E\left[x_{j} F(\mathbf{x}, \mathbf{z})\right]-\omega_{1}^{j} E[F(\mathbf{x}, \mathbf{z})], \\
\dot{\omega}_{11}^{j p} & =E\left[\left(x_{j}-\omega_{1}^{j}\right) A_{p}+\left(x_{p}-\omega_{1}^{p}\right) A_{j}\right. \\
& +E\left[\left(x_{j}-\omega_{1}^{j}\right)\left(x_{p}-\omega_{1}^{p}\right) F(\mathbf{x}, \mathbf{z})\right]-\omega_{11}^{j p} E[F(\mathbf{x}, \mathbf{z})], \\
\dot{\omega}_{2}^{j} & =2 E\left[\left(x_{j}-\omega_{1}^{j}\right) A_{j}(\cdot)\right]+E\left[B_{j j}(\cdot)\right] \\
& +E\left[\left(x_{j}-\omega_{1}^{j}\right)^{2} F(\mathbf{x}, \mathbf{z})\right]-\omega_{2}^{j} E[F(\mathbf{x}, \mathbf{z})]
\end{aligned}
$$

$\forall j, p \in \mathrm{N}_{1}^{7}$, taking into account the above restrictions on the asymptotic stability region. Equations (18), (19) are ordinary differential equations of order 36 with respect to semi-invariants $\omega_{8}^{1}$ and $\omega_{1}^{j}, \omega_{2}^{j}, \omega_{11}^{j p}$ with indexes $j, p \in \mathrm{N}_{1}^{7}$.

\section{The Simulation Results and Discussion}

The dynamics of the UAV turn with a mass of $320 \mathrm{~kg}$ was studied, taking into account wind disturbances caused by atmospheric turbulence. At the flight altitude $H=1000 \quad \mathrm{~m}$ with airspeed $V_{\mathrm{o}}=111 \mathrm{~m} / \mathrm{s}$ and the balancing angle of attack $\alpha_{0}=3.9 \mathrm{deg}$, the UAV aerodynamic coefficients have the following values (Romanenko et al., 2012):

$k_{\beta}=0.1946 ; \quad k_{\gamma}=0.0833 ; \quad l_{\beta}=47.272 ;$

$l_{\omega_{x}}=6.776 ; \quad l_{\omega_{v}}=1.742 ; \quad l_{\mathrm{e}}=176.54 ;$

$n_{\beta}=13.81 ; n_{\omega_{x}}=0.108 ; n_{\omega_{y}}=0.859 ; n_{\mathrm{d}}=7.12$.

Moreover, coefficients of the characteristic polynomial providing stability have the values

$C_{1}=14, C_{1}=69.9, C_{3}=213, C_{4}=217, C_{5}=120$.

In this case, the UAV autopilot ratios are equal to the values

$i_{\beta}^{\mathrm{e}}=-0.268 ; i_{x}^{\mathrm{e}}=0.0093 ; i_{y}^{\mathrm{e}}=0.611 ; q_{\mathrm{e}}=0.58 ;$

$i_{\beta}^{\mathrm{d}}=-0.132 ; \quad i_{x}^{\mathrm{d}}=-0.0152 ; \quad i_{y}^{\mathrm{d}}=0.628$,

which correspond to the following coefficients $a_{i}, i \in \mathrm{N}_{1}^{6}$

$a_{1}=L_{x}=8.42 ; \quad a_{2}=L_{y}=-106.2 ;$

$a_{3}=L_{\delta}=102.3 ; \quad a_{4}=N_{\beta}=12.85 ;$

$a_{5}=N_{x}=0 ; \quad a_{6}=N_{y}=5.39$.

The problem of optimization (18), (19) with restrictions at the turbulence scale $L_{w_{x}}=310 \mathrm{~m}$ is solved with the specified height and the RMS value $\sigma_{\omega_{x}}=2.8 \mathrm{~m} / \mathrm{s}$ for turbulence of the wind, which corresponds to the "strong" turbulence according to the European airworthiness standards. As a result of solving the optimization problem with identifying the UAV angular 
velocities by the proposed approach, the coefficients $a_{i}, i \in \mathrm{N}_{1}^{6}$ were obtained with the values

$a_{1}=L_{x}=8.91 ; \quad a_{2}=L_{y}=-109.6 ;$

$a_{3}=L_{\delta}=99.3 ; \quad a_{4}=N_{\beta}=14.125 ;$

$a_{5}=N_{x}=0 ; \quad a_{6}=N_{y}=4.83$,

which correspond to the autopilot gear ratios with the following values:

$i_{\beta}^{\mathrm{e}}=-0.268 ; i_{x}^{\mathrm{e}}=0.0121 ; i_{y}^{\mathrm{e}}=0.631 ; q_{\mathrm{e}}=0.56 ;$

$i_{\beta}^{\mathrm{d}}=0.045 ; \quad i_{x}^{\mathrm{d}}=-0.0152 ; \quad i_{y}^{\mathrm{d}}=0.55$.

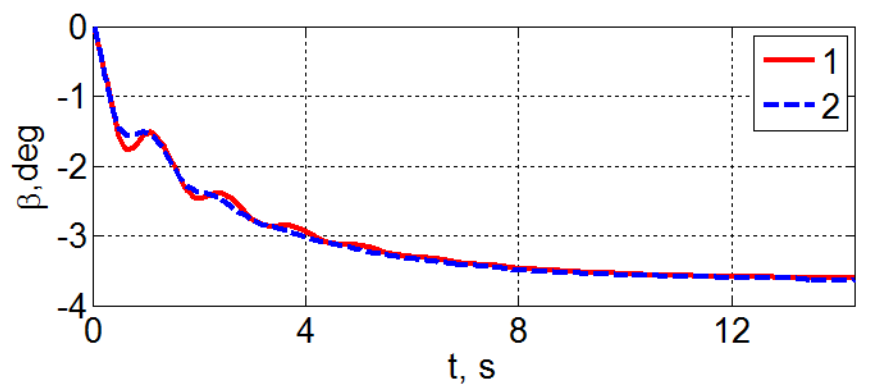

Fig. 5 . The changing average values of angle $\beta$

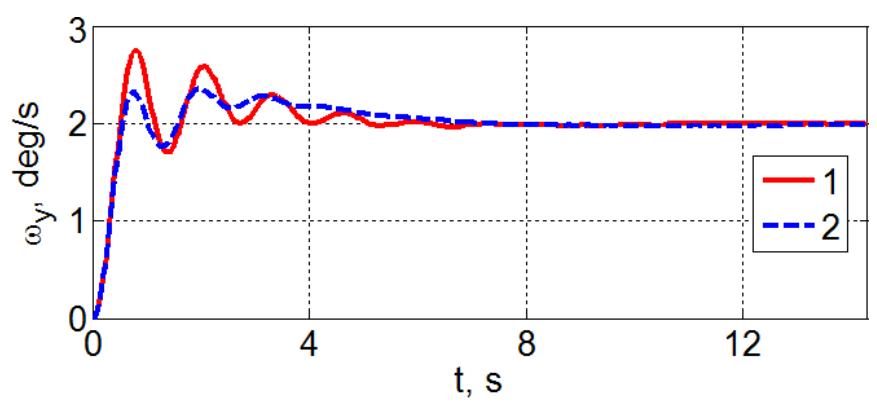

Fig. 6. The changing average values of angular rate

The transients corresponding to the initial values of the autopilot gear ratios (20) are shown in Figs. $5-8$ in continuous graphs $(1, \mathrm{red})$. The transients corresponding to optimized values of the autopilot gear ratios (21), taking into account the wind turbulent disturbances and identification of the angular velocities, $\omega_{x}, \omega_{y}$ are presented in the same figures by dashed graphs $(2$, blue).

The presented data clearly demonstrate that the UAV autopilot parameters synthesized, taking into account wind effects, on average, provide a parry of disturbances caused by atmospheric turbulence, and reduce the amplitude of damped oscillations in the transient processes.

\section{Conclusions}

Elaborated methods for identification of the parameters and control functions of nonlinear stochastic systems with perturbations, noises, and functional equality type constraints are presented. Important applications relating to optimizing the parameters of the UAV autopilot during its rotational maneuver in translational motion when the turbulent wind disturbances, taking into account the identification of the UAV angular velocities, are briefly represented. The article's main breakthroughs are as follows:

(i) For random controlled processes, a fast calculation of semi-invariants is performed with the necessary accuracy. The results are applied for recurrent parametric optimization on the specified criteria;

(ii) The developed algorithms were implemented in contemporary computer-aided technology of designing UAVs.

\section{Abbreviations}

\begin{tabular}{lll}
\hline RMS & $:$ & Root Mean Square \\
SRM & & Space Robot-manipulator \\
UAV & $:$ & Unmanned Aerial Vehicle
\end{tabular}

\section{Funding}

The work was supported by Russian Foundation for Basic Research (RFBR), Grant no. 20-08-00779.

\section{CRediT Author Statement}

Yevgeny Somov: Writing-Original draft preparation, Editing. Nikolay Rodnishchev: Conceptualization, Validation. Tatyana Somova: Software, Simulation, Data Processing, Graphical Representation of Results.

\section{References}

Bodner, V., Rodnishchev, N. and Yurikov, E., 1987, Optimization of Terminal Stochastic Systems. Moscow: Mashinostroyeniye.

Bulinsky, A. V. and Shiryaev, A. N., 2005, Theory of Random Processes. Moscow: PhysMathLit.

Chernetsky, V. I., 1968, Analysis of Accuracy of Nonlinear Control Systems. Moscow: Mashinostroyeniye.

Dashevskii, M.L., 1976, Implementing a moment semiinvariant method for random process analysis. Avtomatika i Telemekhanika, (10), pp.23-26.

Denisov, K. and Rodnishchev, N., 2017, Identification of Parameters and Control for Stochastic Dynamical Systems with Functional Limitations. Kazan: Shkola. 
Dostupov, B. G., Ed., 1970, Statistical Methods in Design of Nonlinear Automatic Control Systems, Moscow: Mashinostroyeniye.

Dubovitskii, A. and Milyutin, A., 1965, Extreme problems with restrictions, USSR Comp. Math. and Math. Phys., 5(3), pp. 1-80.

Evlanov, E. and Konstantinov, V., 1976, Systems with Random Parameters, Moscow: Nauka.

Fleming, W.H. and Rishel, R.W., 2012, Deterministic and stochastic optimal control (Vol. 1), Springer Science \& Business Media.

Gikhman, I.I. and Skorokhod, A.V., 2004, The theory of stochastic processes II, Springer Science \& Business Media.

Girsanov, I.V., 2012, Lectures on mathematical theory of extremum problems (Vol. 67), Springer Science \& Business Media.

Ioffe, A. and Tikhomirov, V., 1974, Theory of Extreme Problems, Moscow: Nauka.

Kazakov, I., 1977, Statistical Dynamics of Systems with Variable Structure, Moscow: Nauka.

Kazakov, I. and Artemyev, V., 1980, Optimization of Dynamic Systems with Random Structure, Moscow: Nauka.

Kolosov, G., 1984, Synthesis of Optimal Automatic Systems at Random Disturbances, Moscow: Nauka.

Kozhevnikov, Yu., 1978, Statistical Optimization of Flying Vehicles, Moscow: Mashinostroyeniye.

Kozhevnikov, Yu., Agdamov, R. et al., 1989, Computer Aided Tests in Aviation Industry, Moscow: Mashinostroyeniye.

Malakhov, A., 1978, Cumulative Analysis of Random nonGaussian Processes and their Transformations, Moscow: Sov. Radio.

Oksendal, B., 2000, Stochastic Differential Equations, New York: Springer Verlag.

Potseluyev, A. V., 1984, Statistical Analysis and Synthesis of Complex Dynamic Systems, Moscow: Mashinostroyeniye.

Pugachev, V. and Sinitsyn, I., 1985, Stochastic Differential Systems, Moscow: Nauka.

Romanenko, L., Samarova, G. and Zaitsev, S., 2012, Control of lateral motion of a flying vehicle with one symmetry plane, Vestnik of Tupolev Kazan State Technical University, (2), pp. 260-267.

Rodnishchev, N., 2001a, Approximate method for optimal control of nonlinear stochastic systems with limitations, Automat. Rem. Contr., 62(2), pp. 248-259.
Rodnishchev, N., 2001b, Necessary conditions for the optimal control of constrained discontinuous nonlinear stochastic systems, J. Comput. Sys. Sc. Int., 40(6), pp. 878-889.

Rodnishchev, N.E. and Khairullin, A.K., 2010, Necessary conditions for optimality of stochastic systems with uncontrollable flow of failures and constraints of the equality and inequality type, Automation and Remote Control, 71(11), pp.2367-2382.

Rodnishchev, N., Somov, Y., Butyrin, S. and Somova, T., 2019, Reliable Control Optimization in Aerospace Engineering at Stochastic Stream of Faults, IFACPapersOnLine, 52(12), pp.188-193.

Rodnishchev, N. and Somova, T., 2019, June. Identification and Stochastic Checking a Control Safety at a Landing of Aerospace Vehicles. In 2019 IEEE 5th International Workshop on Metrology for AeroSpace (MetroAeroSpace) (pp. 506-510), IEEE.

Solodov, A. and Solodov, A., 1988, Statistical Dynamics of Systems with Dotted Processes, Moscow: Nauka.

Somov, Y., Butyrin, S., Somov, S. and Somova, T., 2019, Control of robot-manipulator during its preparation and capture of a passive satellite, Mathematics in Engineering, Science \& Aerospace (MESA), 10(3). 